\title{
Management Issues of HIV Infection in Poor Resource Setting Due to Unavailability of Drug Sensitivity Testing
}

\begin{abstract}
Ahmadu BU
Dr. Baba Usman Ahmadu, (MBBS, MHPM, FMCPaed), Consultant Paediatrician, Department of Paediatrics, Federal Medical Centre (FMC) Yola, Adamawa state, Nigeria; Visiting Consultant Paediatrician, Abubakar Tafawa Balewa University Teaching Hospital (ATBUTH), Bauchi and FMC Jalingo, Taraba state, Nigeria. Formerly of the Department of Paediatrics, University of Maiduguri Teaching Hospital (UMTH), Borno State, Nigeria.
\end{abstract}

\section{Introduction}

$\Delta$ ppearance of new or recurrent clinical Afeatures classified under the World Health Organization (WHO) clinical staging suggests Human Immunodeficiency Virus (HIV) progression in a child on antiretroviral therapy (ART) ${ }^{1}$. Therefore, HIV treatment failure should be considered when either new or recurrent stage 3 or 4 clinical events emerged on a child receiving ART. High mutation rate of HIV severely impairs the effectiveness of highly active anti-retroviral therapy (HAART), define as the combination of at least three antiretroviral (ARV) drugs from more than one class $^{1,2}$. Studies have shown that failure to reduce viral load to below 50 copies $/ \mathrm{ml}$ favors the emergence of HIV drug resistance (HIVDR), and this has been found to be the common cause of treatment failure among HIV cases $^{1,2}$. Investigators recently have reported a remarkable scale-up of antiretroviral therapy (ART) by up to $20 \%$ especially in resource poor countries of the world ${ }^{2,3}$. The number of individuals receiving ART has risen from 6.6 million in 2010 to more than 8 million in $2011^{3}$. Standardized protocol and guidelines for starting and monitoring of ART were some of the reasons given for the increased utilization of HIV drugs. Nonetheless, the existence of resistance HIV strain may diminish the effectiveness of ART. Thus, the physician is saddled with the option of switching to another regimen. Yudong et $\mathrm{al}^{4}$ in 2007 in Canada have found that alterations of HIV receptor through some modification changes led to HIVDR.

\author{
Address for correspondence \\ Dr. Baba Usman Ahmadu \\ E-mail: ahmadu4u2003@yahoo.com
}

\begin{abstract}
Despite the obvious benefits that rapid scale-up of antiretroviral therapy (ART) has had on cases of human immunodeficiency virus (HIV), treatment failure that is mostly associated with the emergence of HIV drug resistance still remains a major challenge. Index case is a stage 4 HIV patient who failed multiple ART, including nucleoside and nonnucleoside reverse transcriptase inhibitors (NRTIs and NNRTIs) and protease inhibitors (PIs). His clinical and immunological markers continued to fail despite completion of anti tuberculosis drugs, adequate nutrition, and treatment for opportunistic infections. This case indicates an emerging need to consider providing facility for HIV drug sensitivity testing at various sites in order to select appropriate second line antiretrovirals that would be of benefit to a patient.
\end{abstract}

Key words: Drug sensitivity testing, HIV Treatment failure, Health resource poor setting.

While a single mutation could be associated with resistance to nevirapine (NVP) and lamuvudine (3TC), several mutations are needed before resistance develops for Zidovudine (AZT) ${ }^{1,2,3,4}$. Because of this, testing for HIVDR is now becoming the standard of care especially where failure of first line ART is suspected ${ }^{2}$. Coalition of agencies known as HIV research network (HIVResNet) has published two types of HIVDR tests; these are genotypic and phenotypic tests ${ }^{2}$. The former predicts the virus susceptibility from mutations identified in the HIV genome and the latter measures the virus susceptibility in various concentrations of the drug. From the aforesaid, switch of HIV drug regimen where failure of the initial first line ART was suspected could be successful if being guided by genotypic or phenotypic testing.

Here, I report on a stage 4 HIV patient who failed multiple ART, including nucleoside and non-nucleoside reverse transcriptase inhibitors (NRTIs and NNRTIs) and protease inhibitors (PIs) at the Federal Medical Center Yola, North-Eastern Nigeria.

\section{How to cite this article?}

Ahmadu BU. Management Issues of HIV Infection in Poor Resource Setting Due to Unavailability of Drug Sensitivity Testing. J Nepal Paediatr Soc 2013;33(2):141-143. 


\section{The Case}

An eight year old boy who is a known HIV-1 stage 4 disease patient presented with chronic history of fever, cough, progressive weight loss, florid oral thrush and diarhoea despite adherence on HAART for three years. Alopecia, severe wasting $10.5 \mathrm{~kg}$ as against $28 \mathrm{~kg}$, which is less than minus two standard deviation (-2SD) from normal for his age and sex, in addition to scaphoid abdomen and persistent generalized lymphadenopathy were observed. His CD4+ T-lymphocyte counts declined from $911 \mathrm{cell} / \mathrm{cu} \mathrm{mm}$ to $38 \mathrm{cell} / \mathrm{cu} \mathrm{mm}$ and $28 \mathrm{cell} /$ $\mathrm{cu} \mathrm{mm}$ respectively within the span of a year. Chest radiograph, manthoux test and blood culture were unremarkable and lack of facility for viral load hindered us from doing the test. He also had hyponatremia of 110 $\mathrm{mmol} / \mathrm{l}$. Diagnosis of HIV co-infected with tuberculosis (TB) to exclude failure of first line ART was made. Non availability of facility for HIV drug sensitivity testing constrained us from excluding first line ART failure, even as the patient's clinical and immunological variables continued to fail despite having had appropriate anti TB, and ART. Adequate nutrition, treatment for opportunistic infections (Ols) and electrolyte correction were also prescribed. The patient was initially on zidovudine (AZT), lamuvudine (3TC), and nevirapine (NVP), which are the first line ART in our setting. Efavirenz (EFZ) substituted NVP at the commencement of anti TB and kaletra (LPV/r), a protease inhibitor and a second line ART was further used in place of EFV on completion of anti TB regimen without significant improvement.

\section{Discussion}

For treatment failure to be considered in a child with HIV, the child should have received HAART for at least 24 weeks, adherence to therapy should be optimal, opportunistic infections should have been treated, and immune reconstitution inflammatory syndrome (IRIS) excluded $^{1}$. Furthermore, the issue of poor growth should be addressed by ensuring that the child is receiving adequate nutrition ${ }^{1}$. Index patient had good adherence on HAART for three years and intercurrent opportunistic infections were treated in addition to adequate dietary provision and supportive care. Still his CD4+ T-lymphocyte count was steadily declining thereby making IRIS a remote consideration, more so, IRIS is less frequently seen in children ${ }^{5}$. By detecting severe recurrent oral candidiasis and a very low CD4+ T-lymphocyte count, the current case could be tagged as having both clinical and immunological definition of HIV treatment failure. This conformed to findings by other workers in which clinical criteria supported by CD4 criteria were labeled as good indicators for identifying HIV treatment failure ${ }^{1,6}$.

For instance immunological treatment failure can be identified by comparing baseline CD4+ T-lymphocyte count, with that obtained from initial immunological response to HAART. A drop in CD4 values below agerelated CD4 threshold at the commencement of HAART after initial immune recovery following HAART could be termed treatment failure. Our present case was commenced on HAART based on HIV stage 4 disease and CD4+ T-lymphocyte count of 300 cells $/ \mathrm{mm}^{3}$, a little less than his threshold of 350 cells/cu mm. After his initiation on HAART, his CD4+ T-lymphocyte count rose to 911 cells/cu mm before a dip to a very low level of 28 cells/cu $\mathrm{mm}$. Thus recognition of treatment failure on the basis of immunological values relies on comparison with previous CD4 values as demonstrated in recent case.

Researchers have documented if CD4+ T-lymphocyte values fall to below 15\% (12-35 months of age), $10 \%$ (36-59 months of age), or $100 \mathrm{cells} / \mathrm{cu}$ $\mathrm{mm}$ ( $>5$ years of age), then the clinician should consider switching ART regimen ${ }^{1,2,6}$. The present case is an eight year old with a CD4+ T-lymphocyte value of value of 28 cells/cu mm, thus the switch in his ART regimen is justifiable. Of note is that immunological criteria for recognizing treatment failure of HIV are supplemental to clinical criteria, because new or recurrent stage three and four events are sufficient for a paediatrician to consider switching regimen as was done in our patient $t^{1,2,6}$. However, it is recommended that a child with TB, or with severe recurrent bacterial pneumonia all of which are considered clinical stage three events should receive appropriate therapy and re-evaluation to determine the need to switch ART before switching ${ }^{1,2,6}$. All these were considered in present case; he became eligible for anti TB therapy at a point and was given full course of treatment.

Even though viral load (VL) is equally important in monitoring patients on HAART, its place in decision making on treatment failure is arguable. While some authors have used it where conflict exists between CD4 and clinical criteria in making the diagnosis of treatment failure, others have found it inefficient in children ${ }^{7,8}$. The VL in our patient was not determined and this is because our HIV service providers have not been doing it as routine. Lack of standard HIV threshold for which treatment failure is define in children could be one reason why $\mathrm{VL}$ is not done by our service providers; despite un-validated report that HIV-RNA values greater than 100,000 copies indicate the need to switch therapy ${ }^{7,8}$. Because total lymphocyte count (TLC) has only been useful in the absence of CD4 measurement in initiating HIV therapy, its poor predictor of treatment success makes it unsuitable for diagnosing treatment failure ${ }^{9}$.

In the event of a change from a first-line to a second-line regimen as was done in index case, the second-line regimen should include new drugs one or 
more from a new class in order to maximize treatment success, based on drugs expected to retain activity against the child's virus ${ }^{1,2,6}$. In order to have second-line regimen that have activity on child's virus, then there is the need for drug sensitivity testing. However, this test may not be feasible in resource-limited settings partly due to the huge financial resource that may be required. It is therefore important to develop and implement less costly, less complex and standardized methods of ART switching. In this vein the WHO drafted guidelines on the use of second-line regimen to be used by countries of the world ${ }^{1,2,6}$. For a patient on standard first line regimen (AZT, 3TC, NVP/EFV), like the current case, at least one of the NRTI should be replaced and the new regimen should be boosted by a protease inhibitor.

For this to be achieved, didanosine (DDI) should be used in place of AZT and lopinavir/ritonavir (LPV/r) should replace NVP/EFV while 3TC is retained. Retention of $3 \mathrm{TC}$ is because its mutation on viral protein M184V confers increased susceptibility of HIV to NRTI, leading to decreased viral fitness $s^{1,2,3,6}$. Placing our patient on this second line protocol was a challenge, because of non availability of DDL in our site. Other NRTI like abacavir $(A B C)$ could also be effective; however, its supply has been erratic in our center. Logically, stavudine (d4T) could also be used, but given the cross-resistance that exists between $\mathrm{d} 4 \mathrm{~T}$ and $\mathrm{AZT}$ coupled with its side effects,

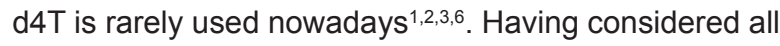
this difficulties the index patient was continued on AZT and 3TC, however, LPV/r was used in place of NVP.

Having acknowledged some of the difficulties encountered in managing advanced cases of HIV chief among them is the lack of drug testing facility; the HIVResNet developed a global frame work for the prevention of treatment failure ${ }^{3}$. These included: 1) Formation of national HIVDR working groups in countries scaling-up ART, 2) Monitoring the quality of care in ART programmes using Early Warning Indicators of HIVDR, 3) Surveillance of acquired HIVDR at sentinel ART clinics, 4) Surveillance of transmitted drug resistant HIV in recently infected populations, 5) Designation, by national HIVDR working groups, of one or more HIVDR testing (genotyping) laboratories for HIVDR surveillance, and 6) Formation and maintenance of a national HIVDR database. The frame work included the development of a network of HIVDR genotyping laboratories that support public health surveillance. Practically, these components are still not fully explored in our center and other centers from low resource setting, possibly because of the cost implications.

\section{Conclusion}

Children placed on ART could also develop HIV treatment failure that is caused mainly by HIVDR. As such policy makers should ensure adherence to HIV guidelines and adequate supply of first and second line HAART regimen in order to avoid disease progression. In addition, HIV sites should have drug testing facility in order to select appropriate second line ARV that would be of benefit to a patient.

Acknowledgement: I would like to thank Dr. J Sharah for his technical assistance.

\section{References}

1. Antiretroviral therapy of HIV infection in infants and children: towards universal access recommendations for a public health approach. World Health Organization, 2006. Available at: http://www.who.int/hiv/pub/guidelines/en.

2. World Health Organization HIV Drug Resistance Global Report, 2012. Available at: http://www.who. int/hiv/pub/drugresistance/report2012/en/index. html.

3. Joint United Nations Programme on HIV/ AIDS. Together we will end AIDS. (Update 2012). Available at: http://www.unaids. org/en/media/unaids/contentassets / documents/epidemiology/2012/20120718_ togetherwewillendaids_en.pdf.

4. Yudong Q, Bluma GB, André D, et al. Highly diversified multiply drug-resistant HIV-1 quasispecies in PBMCs: a case report. Retrovirology 2008;5:43-9.

5. Hirsch $H H$, Kaufmann $G$, Sendi $P$, et al. Immune reconstitution in HIV-infected patients. Clin Infect Dis 2004;38:1159-66.

6. World Health Organization. Scaling up antiretroviral therapy in resource-limited settings: treatment guidelines for a public health approach. 2003 revision. Geneva, (www.who.int/hiv).

7. Dunn D. Short-term risk of disease progression in HIV-1-infected children receiving no antiretroviral therapy or zidovudine monotherapy: a metaanalysis. Lancet 2003;362:1605-11.

8. Mofenson LM, Korelitz J, Meyer WA, et al. The relationship between serum human immunodeficiency virus type 1 (HIV-1) RNA level, CD4 lymphocyte percent, and long term mortality risk in HIV-1-infected children. National Institute of Child Health and Human Development Intravenous Immunoglobulin Clinical Trial Study Group. J Infect Dis 1997;175:1029-38.

9. Florence E, Dreezen C, Schrooten W, et al. The role of non-viral load surrogate markers in HIV-positive patient monitoring during antiviral treatment. Int $J$ STD AIDS 2004;15:538-42. 